\title{
Độ NHẠY CỦA MỘT SỐ CHỈ SỐ KHÔ HẠN Ở KHU VỰC PHÍA NAM VIẸTT NAM
}

\author{
LƯƠNG VĂN VIÊTT \\ Viện Khoa học Công nghệ và Quản lý Môi truờng, Đại học Công nghiệp Thành phố Hồ Chí Minh; \\ luongvanviet@iuh.edu.vn
}

Tóm tắt.Nghiên cứu này nhằm đánh giá độ nhạy và mức độ phù hợp của một số chỉ số khô hạn đối với các tỉnh phía nam Việt Nam (SVN). Có 3 nhóm chỉ số khô hạn được đưa vào phân tích là nhóm các chỉ số thể hiện sự biến động của lượng mưa, nhóm các chỉ số thể hiện cân bằng giữa mưa với bốc hơi và nhóm còn lại thể hiện biến động của cặp nhiệt độ cùng với lượng mưa. Kết quả nghiên cứu cho thấy với các chỉ số khô hạn được xây dựng dựa trên 1 yếu tố là lượng mưa thì Chỉ số phần trăm lượng mưa tính theo giá trị trung bình $(\mathrm{PN})$ là một chỉ số thể hiện được tình hình khô hạn khu vực và có quan hệ tốt với Chỉ số Niño đại dương $(\mathrm{ONI})$. Với các chỉ số khô hạn được xây dựng dựa trên lượng mưa và lượng bốc thoát hơi thì Chỉ số khô cằn $(\mathrm{AIu})$ là thích hợp cho việc lựa chọn vì nó khá phù hợp với tình hình khô hạn khu vực nghiên cứu và cũng có quan hệ tốt với ONI. Đối với chỉ số khô hạn dựa trên nhiệt độ và lượng mưa, chỉ số Ped thể hiện rõ nét nhất các ảnh hưởng của ENSO.Tuy nhiên Ped chỉ phù hợp khi phân tích tình hình khô hạn rời rạc cho từng thời gian trong năm.

Từ khóa: Chỉ số khô hạn, vùng khí hậu, El Niño, ENSO.

\section{SENSITIVITY OF SOME DROUGHT INDICIES IN SOUTH VIETNAM}

\begin{abstract}
This study aimed to assess the sensitivity and suitability of some drought indices for southern Vietnam (SVN). Three groups of drought indexes were included in the analysis, including one group consisting of only one factor is rainfall, a group of indicators showing the balance between rainfall and evaporation, and the other group showing fluctuations of temperature and amount of rain. The study results show that with the drought index based on rainfall, the Percent of Normal Index (PN) showed the drought condition of the study basin more realistically than other drought Indicators and have a good relationship with the Ocean Niño Index (ONI). For drought indices based on rainfall and evaporation, the Aridity Index (AIu) is an option because it is quite suitable for the drought situation in the study area and also has a good relationship with ONI. For drought index based on temperature and rainfall, Ped shows most clearly the effects of ENSO. However, this indicator is only suitable for analyzing drought situation at discrete times. In addition, it is necessary to adjust the break values on drought classification for PN, AIu and Ped to better suit the climate type of this area.
\end{abstract}

Key words: Drought index, climatic region, El Niño, ENSO.

\section{1. ĐẠT VẤN ĐỀ}

ENSO là tên viết tắt để chỉ sự xuất hiện đồng thời của hai hiện tượng là El Niño, La Niña và dao động Nam (Southern Osillation - SO). Khi El Niño hoạt động thì gây ra các dị thường đáng kể của nhiệt độ, lượng mưa, độ ẩm, số giờ nắng và làm hạn hán xuất hiện ở nhiều nơi. Khu vực nghiên cứu là $\mathrm{SVN}$, bao gồm 3 vùng là: (1) Ven biển phía nam South Central Coast, $\mathrm{RG}_{1}$; (2) Tây Nguyên, $\mathrm{RG}_{2}$; và (3) Nam bộ, vùng này gồm Đồng bằng sông Cửu Long và Đông Nam Bộ, RG 3 . Theo Lương (2006), Chen (2012), Nguyen (2014), Gobin (2015), Ha (2016), Mai (2017), Nguyen (2019), Holly (2019), SVN nằm trong vùng nhiệt đới gió mùa, với vĩ độ từ $8^{\circ} \mathrm{N}-16^{\circ} \mathrm{N}$ và có bờ biển dài nên các ảnh hưởng của $\mathrm{ENSO}$ đến khu vực này là rất rõ nét. Trong các đợt $\mathrm{El}$ Niño cường độ mạnh, tình hình nắng nóng và khô hạn trên khu vực này là rất nghiêm trọng. Điển hình là trong đợr El Niño mạnh kéo dài từ tháng 11/2014 đến tháng 5/2016, khu vực Nam Trung bộ, Tây Nguyên và miền nam Việt Nam đã trải qua hạn hán và xâm nhập mặn tồi tệ nhất trong 90 năm qua. Theo số liệu từ tổng cục thống kề Việt Nam trong thông báo về "Tình hình kinh tế - xã hội 4 tháng đầu năm 2016", đến thời điểm 24/4/2016, đã có 15 tỉnh công bố tình trạng thiên tai hạn hán, xâm nhập mặn. Trong đó, 6 tỉnh công bố thiên tai hạn hán: Gia Lai, Bình Thuận, Ninh Thuận, Kon Tum, Đắk Lắk và Đắk Nông; 6 tỉnh công bố thiên tai xâm nhập mặn: Bến Tre, Cà Mau, Kiên Giang, Trà Vinh, Tiền Giang và Long An; 
3 tỉnh công bố thiên tai hạn hán và xâm nhập mặn: Vĩnh Long, Sóc Trăng và Bạc Liêu. Tính đến thời điểm này có 424,1 nghìn hộ thiếu nước sinh hoạt; 248,3 nghìn ha lúa, 18,9 nghìn ha hoa màu, 53,8 nghìn ha cây ăn quả, 105,2 nghìn ha cây công nghiệp và 5,7 nghìn ha diện tích nuôi trồng thủy sản bị hư hỏng. Tổng giá trị thiệt hại ước tính trong bốn tháng đầu năm 2016 gần 6,4 nghìn tỷ Việt Nam đồng, khoảng 300 triệu đô. Việc đánh giá hoặc cảnh báo khô hạn thường được thông qua các chỉ số khô hạn. Để thấy được độ nhạy cũng như mức độ phù hợp của các chỉ số khô hạn với SVN, dưới đây sẽ phân tích 9 chỉ số hạn được nhiều quốc gia sử dụng.

\section{SỐ LIẾU VÀ PHƯƠNG PHÁP NGHIÊN CÚU}

\subsection{Các chỉ số khô hạn sử dụng trong nghiên cứu}

Đặc điểm của hạn hán là khác nhau ở các vùng khí hậu khác nhau và tác động của nó phụ thuộc vào đặc điểm môi trường và kinh tế xã hội địa phương. Theo Tổ chức Khí tượng Thế giới (WMO, 2016), không có định nghĩa về hạn hán nào hoặc một chỉ số hạn hán nào có thể giải thích và áp dụng cho tất cả các loại hạn hán, chế độ khí hậu và các ngành bị ảnh hưởng bởi hạn hán.

Các chỉ số hạn hán được chọn phân tích trong nghiên cứu này bao gồm chỉ số SPI (the Standard Precipitation Index), CZI (the China-Z index), Z-Score, EDI (the Effective Drought Index), PNI (the Percent of Normal Index), SPEI (the Standard Precipitation Evapotranspiration Index), AIu (Aridity Index) và Ped. Trong số 8 chỉ số này có 5 chỉ số được xây dựng dựa trên 1 yếu tố là lượng mưa gồm: SPI, Z-Score, CZI, PNI và EDI. Các chỉ số được xây dựng dựa trên lượng mưa và bốc thoát hơi là SPEI và Aiu. Chỉ số còn lại là Ped, chỉ số này liên quan đến nhiệt độ và lượng mưa. Để phù hợp với khu vực nghiên cứu, bước thời gian xây dựng các chỉ số này được lựa chọn là 3 tháng. Với bước thời gian này còn thể hiện được sự thay đổi theo mùa của một số các chỉ số và cho kết quả ổn định. Thang phân loại hạn hán được trình bày trong Bảng 1 .

Bảng 1. Phân loại tình hình khô hạn theo các chỉ số hạn

\begin{tabular}{lcccc}
\hline \multicolumn{1}{c}{ Cấp } & $\begin{array}{c}\text { SPI, Z-Score, } \\
\text { CZI, EDI, SPEI }\end{array}$ & PNI (\%) & AIu & Ped \\
\hline Hết sức ẩm ướt & $\geq 2$ & & & \\
Rất âm ướt & 1,5 to 1,99 & & & \\
Ấm ướt nhẹ & 1,0 to 1,49 & $\geq 110^{*}$ & $\geq 0,65^{*}$ & $\geq 110^{*}$ \\
Bình thường & $-0,99$ to 0,99 & 80 to 110 & 0,5 to $0,65^{*}$ & 80 to 110 \\
Khô hạn nhẹ & $-1,0$ to $-1,49$ & 55 to 80 & 0,2 to 0,5 & 55 to 80 \\
Khô hạn nặng & $-1,5$ to $-1,99$ & 40 to 55 & 0,05 to 0,2 & 40 to 55 \\
Hết sức khô hạn & $\leq-2$ & $\leq 40$ & $<, 05$ & $\leq 40$ \\
\hline
\end{tabular}

Ngoài 8 chỉ số hạn trên, chỉ số hạn K của Việt Nam cũng được sử dụng để so sánh.

\section{- SPI}

SPI được McKee cùng các đồng sự đề xuất vào năm 1993, đây là một chỉ số được sử dụng rộng rãi trên thế giới. SPI là một chỉ sô được xác định dựa trên hàm phân bố của lượng mưa và được tính với các bước thời gian khác nhau, từ 1 đến nhiều tháng. Gọi $x$ là lượng mưa ứng với bước thời gian chọn trước của một tháng bất kỳ trong năm, SPI sẽ được tính theo các bước sau:

+ Xác định các tham số hình dạng shapeparameter $(\beta)$ và tham số tỷ lệ scaleparameter $(\alpha)$ theo The Gamma distribution như sau:

$$
\begin{aligned}
& \beta=\frac{1+\sqrt{1+4 U / 3}}{4 U}, \\
& \alpha=\frac{\bar{X}}{\beta},
\end{aligned}
$$

ở đây $\bar{X}$ là giá trị trung bình của $\mathrm{X}$ và $\mathrm{U}$ là hệ số thống kê. Called $\mathrm{n}$ is the number of precipitation observations, khi đó $U$ được tính như sau:

$$
U=\ln (\bar{X})-\frac{\sum \ln (X)}{n}
$$


+ Xác định hàm phân bố Gamma theo phương trình sau:

$$
G(x)=\frac{\int_{0}^{x} x^{\alpha-1} e^{\frac{-x}{\beta}} d x}{\beta^{\alpha} \Gamma(\alpha)},
$$

ở đây $\Gamma(\alpha)=(\alpha-1)$ !, nó được gọi là hàm Gamma. Vì hàm Gamma không được xác định cho $\mathrm{x}=0$ và phân phối lượng mưa có thể chứa các số không, nên xác suất tích lũy trở thành:

$$
H(x)=q+(1-q) G(x),
$$

trong đó $\mathrm{q}$ là xác xuất ứng với $\mathrm{x}=0$.

+ Tính SPI

Xác suất tích lũy $\mathrm{H}(\mathrm{x})$ sau đó được chuyển thành biến ngẫu nhiên chuẩn hóa với giá trị trung bình bằng 0 và phương sai bằng một, là giá trị của $\mathrm{SPI}$ :

Ở đây

$$
\begin{aligned}
& S P I=\frac{2,515517+0,802583 t+0,010328 t^{2}}{1+1,432788 t+0,189269 t^{2}+0,001308 t^{3}}-t \quad 0<H(x) \leq 0,5 \\
& S P I=t-\frac{2,515517+0,802583 t+0,010328 t^{2}}{1+1,432788 t+0,189269 t^{2}+0,001308 t^{3}} \quad 0,5<H(x) \leq 1
\end{aligned}
$$

$$
\begin{aligned}
& t=\sqrt{\ln \left(\frac{1}{H(x)^{2}}\right)} \quad 0<H(x) \leq 0,5 \\
& t=\sqrt{\ln \left(\frac{1}{(1-H(x))^{2}}\right)} \quad 0,5<H(x) \leq 1
\end{aligned}
$$

Để tính SPI, nghiên cứu này sử dụng subroutine cdfgam để tính hàm phân bố Gamma. Subroutine này được lấy trong gói Cdflib.f90 từ trang của Florida State University, https://people.sc.fsu.edu/ jburkardt/f_src/cdflib/cdflib.html.

\section{- CZI và Z-Score}

CZI được sử dụng rộng rãi bởi NCC (National Climate Centre) của Trung Quốc để theo dõi tình trạng hạn hán trên toàn quốc (Wu et al., 2001). Chỉ số này có thể được coi là dữ liệu lượng mưa tuân theo phân phối Pearson Type III và được tính như sau:

$$
\begin{aligned}
& C Z I_{j}=\frac{6}{C_{S}}\left(\frac{C_{S}}{2} \varphi_{j}+1\right)^{1 / 3}-\frac{6}{C_{S}}+\frac{C_{S}}{6}, \\
& C_{s}=\frac{\sum_{j=1}^{n}\left(x_{j}-\bar{x}\right)^{3}}{n \sigma^{3}}, \\
& \varphi_{j}=\frac{x_{j}-\bar{x}}{\sigma},
\end{aligned}
$$

trong đó $\mathrm{j}$ là tháng hiện tại, $\mathrm{Cs}$ là độ lệch, $\mathrm{n}$ là độ dài chuỗi, $\varphi$ là độ lệch chuẩn, còn được gọi là Z-Score (Zsc) và $\mathrm{x}_{\mathrm{j}}$ là lượng mưa của tháng $\mathrm{j}$. Khác với $\mathrm{CZI}$, SPI, không yêu cầu điều chỉnh chỉ số Zsc theo các hàm phân phối Gamma hoặc Pearson loại III.

- EDI

EDI được đề xuất bởi Byun and Wilhite (1996), nó là hàm của PRN (lượng mưa cần thiết để trở về trạng thái bình thường) và được tính như sau:

$$
E D I_{j}=\frac{P R N_{j}}{S T\left(P R N_{j}\right)},
$$


với

$$
\begin{aligned}
& P R N_{j}=\frac{D E P_{j}}{\sum_{k=1}^{j}(1 / k)}, \\
& D E P=E P-M E P,
\end{aligned}
$$

trong đó $\mathrm{j}$ là thời điểm tính toán, $\mathrm{ST}(\mathrm{PRN})$ là độ lệch chuẩn của $\mathrm{PRN}$, EP là lượng mưa hiệu quả và MEP là giá trị trung bình của mỗi ngày $\mathrm{EP}$. Khi bước thời gian được thiết lập, $\mathrm{EP}$ hàng ngày được tính theo phương trình sau:

$$
E P_{i}=\sum_{n=1}^{i}\left[\left(\sum_{m=1}^{n} P_{m}\right) / n\right]
$$

Ở đây $\mathrm{i}$ là khoảng thời gian tính toánvà $\mathrm{P}_{\mathrm{m}}$ là lượng mưa của $\mathrm{m}-1$ ngày trước đó. Dữ liệu lượng mưa để tính EDI có thời gian hàng ngày, nên cần trung bình các giá trị hàng ngày để tính toán EDI hàng tháng. - PNI

Chỉ số PNI được Willeke và các cộng sự xây dựng (1994), là phần trăm của lượng mưa tính trên mức trung bình.

$$
E P N=\frac{P}{\bar{P}} 100 \%
$$

Trong đó $\mathrm{P}$ là lượng mưa của thời điểm tính toán và $\bar{P}$ là lượng mưa trung bình của thời đoạn. Chỉ số PNI rất đơn giản và dễ tính toán và có thể được tính cho nhiều bước thời gian khác nhau.

- SPEI

SPEI là một chỉ số hạn hán được tính dựa trên mức chênh giữa lượng mưa và lượng bốc hơi tiềm năng (Vicente-Serrano et al., 2010). Với bước thời gian tích lũy bất kỳ, gọi $\mathrm{P}_{\mathrm{i}}$ và $\mathrm{ET}_{\mathrm{i}}$ tương ứng là lượng mưa và lượng bốc thoát hơi cho thời điểm $\mathrm{I}$, khi đó mức chênh $\mathrm{D}_{\mathrm{i}}$ được xác định như sau:

$$
D_{i}=P_{i}-E T_{i}
$$

Sau khi tính toán chuỗi Di, hàm phân bố phù hợp nhất cho chuỗi này được ước tính. Xác suất của mỗi giá trị $\mathrm{D}_{\mathrm{i}}$ được tính bằng hàm phân phối tích lũy theo phương trình (17) và được gọi là chỉ số $\mathrm{SPEI}$ (Abramowitz and Stegun, 1965):

$$
S P E I=W-\frac{2,515517+0,802853 W+0,010328 W^{2}}{1+1,432788 W+0,189629 W^{2}+0,001308 W^{3}},
$$

trong đó, $\mathrm{W}=(-2 \ln \mathrm{P})^{0,5}$ cho $\mathrm{P} \leq 0,5$. Nếu $\mathrm{P}>0,5$, thì $\mathrm{P}$ được thay thế bằng $1-\mathrm{P}$ và dấu của $\mathrm{SPEI}$ bị đảo ngược. Thang phân loại SPEI được đưa ra trong Bảng 1. Trong nghiên cứu này, lượng bốc hơi tham chiếu được tính theo phương pháp FAO Penman-Monteith.

- $A I u$

Tổ chức Giáo dục, Khoa học và Văn hóa của Liên Hợp Quốc (UNESCO, 1979) đã đưa ra một chỉ số khô cằn dựa trên tỷ lệ lượng mưa hàng năm $(\mathrm{P})$ so với tỷ lệ bốc hơi tiềm năng $(\mathrm{ET})$, như sau:

$$
A I u=\frac{P}{E T}
$$

Trong nghiên cứu này, phương pháp FAO Penman-Monteith cũng được chọn để tính toán ET. Dữ liệu khí tượng yêu cầu để ước tính ET bằng phương trình FAO Penman-Monteith bao gồm nhiệt độ không khí, độ ẩm không khí, tốc độ gió và bức xạ. AIu được tính với các bước thời gian từ một đến vài tháng.

$-\boldsymbol{K}$

Ngược lại với $\mathrm{AIu}$, chỉ số $\mathrm{K}$ được tính bằng tỷ số giữa lượng bốc hơi tiềm năng và lượng mưa như sau:

$$
K=\frac{E T}{P}
$$

$\mathrm{K}$ được tính với bước thời gian 1 tháng, khi giá trị của $\mathrm{K}$ nằm trong khoảng từ 1 đến 2 và kéo dài trên 2 tháng thì hạn được xem là khô hạn (Bảng 2). So với AIu thì việc xác định hạn theo $\mathrm{K}$ có thể chặt chẽ hơn, 
ngoài giá trị độ lớn của chỉ số còn cần phải xác định thêm thời gian kéo dài.

Bảng 2. Cấp hạn theo K

\begin{tabular}{lcc}
\hline \multicolumn{1}{c}{ Cấp } & Số tháng & $\mathrm{K}$ \\
\hline Bình thường & $<2$ & $<1$ \\
Khô hạn nhẹ̆ & $\geq 2-3$ & $\geq 1-2$ \\
Khô hạn nặng & $\geq 3-5$ & $\geq 2-4$ \\
Hết sức khô hạn & $\geq 5$ & $\geq 4$ \\
\hline
\end{tabular}

- Ped

Chỉ số Ped, được giới thiệu bởi Pedey (1975) và được sử dụng để xác định hạn hán tại Trung tâm Khí tượng Thủy văn của Nga. Ưu điểm của chỉ số này là dễ dàng phân loại điều kiện thời tiết. Công thức được tính như sau:

$$
P e d=\frac{\Delta T}{\sigma_{T}}-\frac{\Delta P}{\sigma_{P}},
$$

ở đây $\Delta \mathrm{T}$ và $\Delta \mathrm{P}$ tương ứng là dị thường nhiệt độ và lượng mưa; $\sigma_{\mathrm{T}}$ và $\sigma_{\mathrm{P}}$ is the standard deviation of the precipitation. So với các chỉ số khác thì chỉ số Ped ngược dấu, chỉ số này thể hiện cả khô hạn và nắng nóng.

\subsection{Số liệu sử dụng}

Các chỉ số giám sát sát ENSO được sử dụng bao gồm ONI (Oceanic Niño Index), chỉ số của JMA (Japan Meteorological Agency), MEI (Multivariate ENSO Index), SOI (Southern Oscillation Index). Trong đó ONI được xây dựng dựa trên SST của khu vực Niño 3,4, chỉ số ENSO của JMA được xây dựng dựa trên SST của khu vực Niño 3, MEI được xây dựa trên 6 biến số khí tượng ở vùng nhiệt đới Thái Bình Dương bao gồm nhiệt độ nước biển bề mặt (Sea Surface Temperature - SST), khí áp bề mặt biển (Sea Level Pressure - SLP), nhiệt độ không khí bề mặt, các thành phần của thành gió kinh tuyến, tổng phần mây của bầu trời; SOI dựa trên SLP của trạm Darwin và Tahiti (Hình 1).

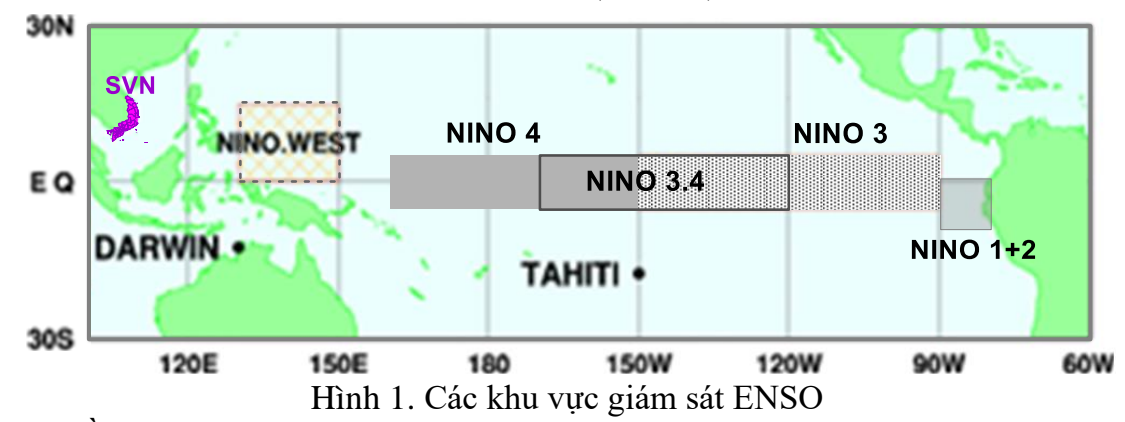

Các pha ENSO bao gồm pha nóng $(\mathrm{W})$, pha trung tính $(\mathrm{N})$ và pha lạnh $(\mathrm{C})$. W và $\mathrm{C}$ tương ứng với các kỳ El Niño và La Niña, các pha này được sử dụng trong phân tích các chỉ số khô hạn. Trung tâm Dự báo Khí hậu Hoa Kỳ $(\mathrm{CPC})$ và Viện Nghiên cứu Quốc tế về Khí hậu và Xã hội (IRI) xác định các giai đoạn ENSO dựa trên ONI, ONI được tính bằng dị thường nhiệt độ nước biển tầng mặt (SSTA) của trung bình trượt 3 tháng khu vực Niño 3,4. Khi ONI đạt trên $+0,5^{\circ} \mathrm{C}$ (nhỏ hơn $-0,5^{\circ} \mathrm{C}$ ) và kéo dài từ 5 tháng liên tục trở lên thì được gọi là kỳ El Niño (La Niña). Cơ quan Khí tượng Nhật Bản (JMA) định nghĩa rằng El Niño (La Niña) sao cho SSTA trung bình trượt 5 tháng đối với Niño 3 liên tiếp bằng $+0,5^{\circ} \mathrm{C}\left(-0,5^{\circ} \mathrm{C}\right)$ hoặc cao hơn (thấp hơn) trong sáu tháng liên tiếp hoặc lâu hơn.

Số liệu khí hậu từ các trạm quan trắc khí tượng trên khu vực nghiên cứu được thể hiện trên Hình 2 . Để thuận lợi cho việc trình bày ở đây sử dụng ID của trạm để nhận diện trạm. Có tổng số 40 trạm quan trắc đưa vào phân tích trong đó $R_{1}$ có 12 trạm với $I D$ từ 1 tới $12, R_{2}$ có 10 trạm với $I D$ từ 13 tới 22 và $R G_{3}$ là 18 trạm với ID từ 23 tới 40. Số liệu được sử dụng là từ năm 1977-2018, 42 năm, khoảng thời gian này được lựa chọn vì số liệu quan trắc nhiệt độ của các trạm thuộc SVN khá ổn định. Số liệu sử dụng gồm nhiệt độ, lượng mưa và các yếu tố khác liển quan đến tính toán bốc hơi tiềm năng như gió, độ ẩm và bức xạ. 


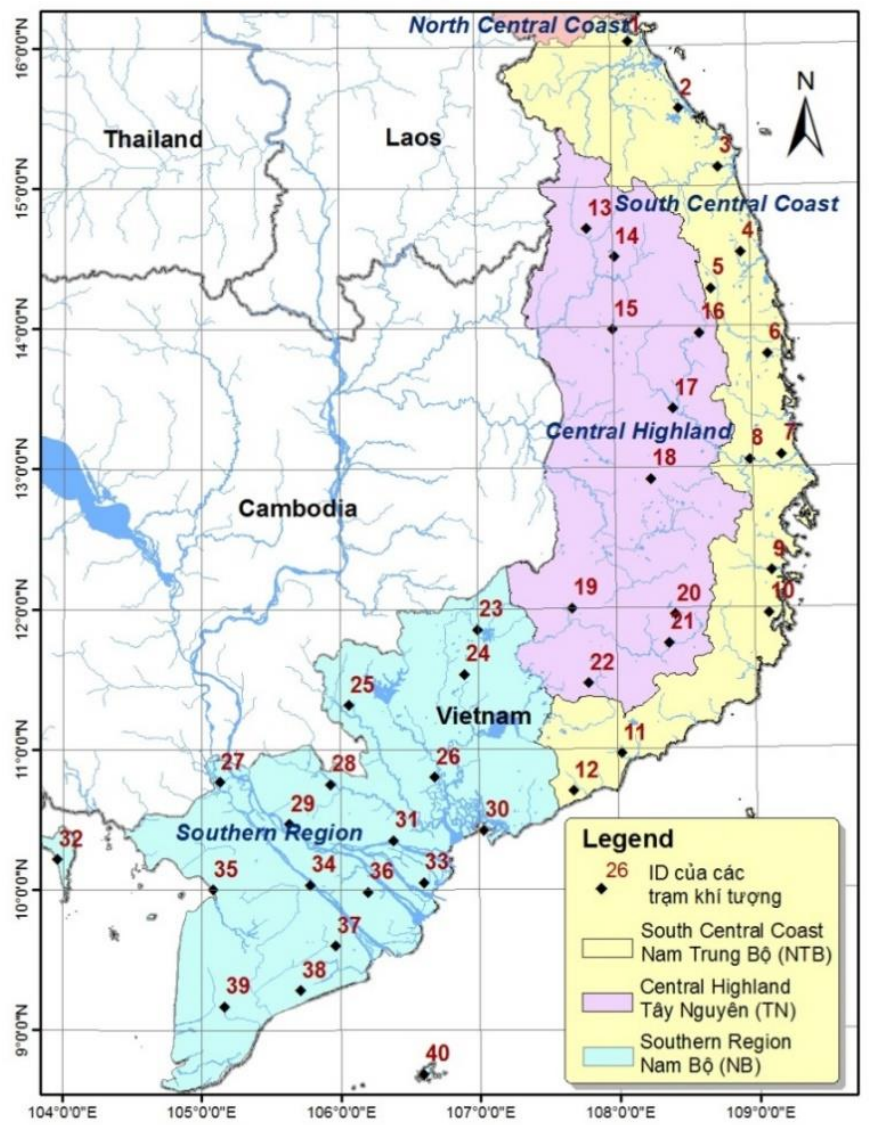

Hình 2. Các trạm khí tượng sử dụng trong nghiên cứu

\section{KẾT QUẢ VÀ THẢO LUẬN}

\section{1. Độ nhậy và sự phù họ̣p của các chỉ số hạn đối với khu vực nghiên cứu}

Để lựa chọn một chỉ số hạn cho khu vực nghiển cứu dưới đây sẽ phân tích để làm rõ độ nhạy của các chỉ số này, và sự phù hợp của các chỉ số với phân bố các yếu tố khí hậu trên khu vực nghiên cứu.

Dựa trên thang phân loại về mức độ hạn hán trên bảng 1 , kết quả xác định thời gian hạn hán theo các cấp tính bằng phần trăm được thể hiện trong bảng 2 . Nếu sử dụng khái niệm về hạn là sự thiếu hụt lượng mưa so với trung bình nhiều năm thì phần lớn các chỉ số về hạn đều thích hợp, nhất là các chỉ số như SPI, CZI. Tuy nhiên nếu sử dụng khái niệm hạn là sự thiếu hụt lượng mưa so với trung bình và lượng mưa không đáp ứng các yêu cầu cho sinh hoạt và sản xuất thì một số các chỉ số trên chưa thực sự phù hợp với khu vực nghiên cứu. Từ bảng 2 cho thấy ngoài các chỉ số $\mathrm{PN}$, AIu và Ped thì thời gian khô hạn tính theo các cấp hạn là khá nhỏ. So với mùa khô trên khu vực nghiên cứu kéo dài khoảng 5 tháng thì các con số này hoàn toàn không phù hợp.

\begin{tabular}{|c|c|c|c|c|c|c|c|c|c|}
\hline \multirow[b]{2}{*}{ Cấp hạn } & \multicolumn{8}{|c|}{ Bảng 3. Thời gian khô hạn theo các cấp hạn (\%) } & \multirow[b]{2}{*}{ Ped } \\
\hline & SPI & Zsc & CZI & EDI & PN & SPEI & $\mathrm{K}$ & AIu & \\
\hline Khô hạn nhẹ & 5,8 & 4,2 & 5,2 & 4,4 & 14,3 & 6,9 & 23,2 & 14,9 & 10,5 \\
\hline Khô hạn nặng & 1,2 & 0,0 & 0,8 & 0,0 & 6,8 & 1,4 & 9,8 & 7,9 & 5,2 \\
\hline $\begin{array}{l}\text { Hết sức khô } \\
\text { hạn }\end{array}$ & 0,0 & 0,0 & 0,0 & 0,0 & 3,8 & 0,4 & 3,7 & 0,8 & 0,0 \\
\hline Tổng cộng & 6,9 & 4,2 & 6,0 & 4,4 & 24,8 & 8,7 & 36,7 & 23,6 & 15,7 \\
\hline
\end{tabular}

Theo bảng 2, mức độ hạn không thể hiện rõ ở nhóm các chỉ số gồm SPI, Zsc, CZI, EDI và SPEI, ở cấp hạn nhẹ thời gian khô hạn cũng chỉ từ 4,2 đến $6,9 \%$. Còn nếu xét ở cấp Hết sức khô hạn thì thời gian xảy ra là gần như không đáng kể. Do 5 chỉ số có cùng thang độ hạn và do cách chuẩn hóa các chỉ số này nên chúng có mối quan hệ khá chặt với nhau và có hệ số tương quan từ 0,898 đến 0,995 , trong đó giá trị thấp nhất thuộc về cặp EDI và SPEI, và giá trị cao nhất thuộc về cặp SPI và CZI. Do đó, tình hình hạn được thể hiện bằng các chỉ số này cho kết quả gần tương tự. Tuy nhiên Zscore và EDI có thời gian hạn ngắn hơn, hay 
mức độ nhạy kém hơn so với SPI (xem Hình 3 và Bảng 3 ). Điều này trái ngược với nhận xét của Vinit và các cộng sự (2015) là EDI phù hợp với thực tế hơn so với các chỉ số SPI, Z-Score, CZI. Hình 3 và Bảng 3 cho thấy trong các chỉ số này thì SPEI có độ nhạy tốt hơn. Ngoài ra SPE còn thể hiện rõ hơn các mức hạn nặng, điều này phù hợp với nhân xét của Ali và các cộng sự (2017) về độ nhạy của SPEI với hạn nặng. Hệ số tương quan giữa SPI và CZI rất cao và kết quả thống kê tình hình hạn cũng giống nhau, $\mathrm{CZI}$ có cách tính đơn giản hơn so với SPI, như vậy CZI là một khuyến nghị cho sử dụng.
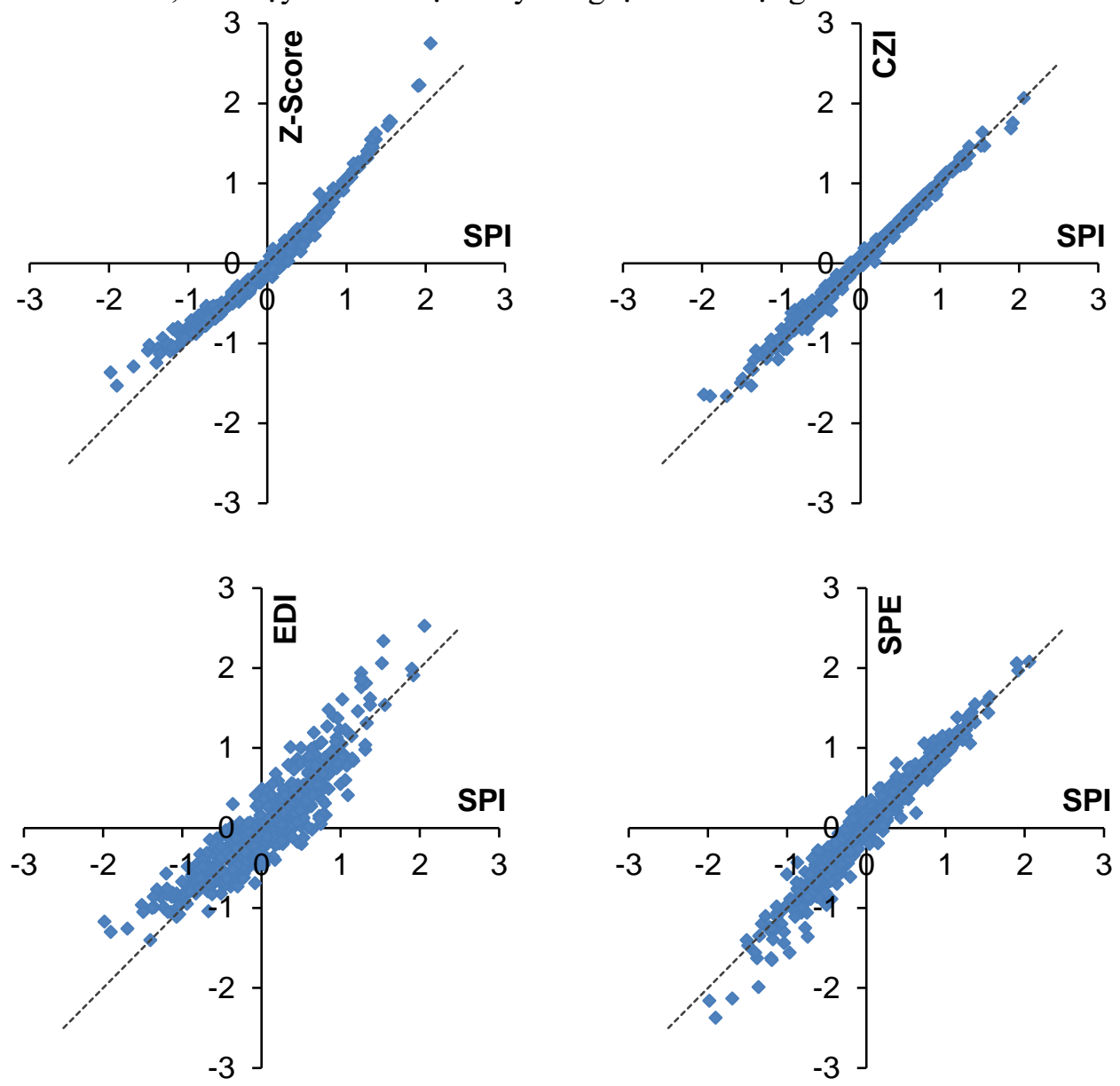

Hình 3. Hệ số tương quan giữa SPI với Z-Score, CZI, EDI và SPEI

Để thấy được khả năng phản ánh hạn hán theo thời gian trong năm của các chỉ số này, kết quả thống kê khả năng xuất hiện theo các tháng trong năm được trình bày trên Hình 4 . Trên hình này, ứng với mỗi chỉ số khô hạn, giá trị của mỗi tháng được tính bằng phần trăm số ngày khô hạn của tháng trên số ngày khô hạn cả năm. Hình này cho thấy ngoại trừ PNI, $\mathrm{K}$ và $\mathrm{AIu}$, các chỉ số còn lại không thể hiện sự khác biệt rõ rệt theo các tháng trong năm. K và AIu thể hiện thời gian hạn xảy ra chủ yếu từ tháng 1 tới tháng 5 , trong khi đó PNI lại thể hiện hạn xảy ra từ tháng 12 tới tháng 5 . Do bước thời gian tích lũy là 3 tháng và mùa khô trên khu vực này là từ 12 tới tháng 5 nên PNI khá phù hợp với tình hình phân bố mưa xảy ra trên khu vực này. Thời gian khô hạn theo chỉ số $\mathrm{K}$ và $\mathrm{AIu}$ xảy ra chủ yếu vào tháng 2 tới tháng 4 là do đây là các tháng trong mùa khô nên lượng mưa khá thấp, ngoài ra do nền nhiệt độ cao nên đây cũng là các tháng có ET lớn. Ngoài $\mathrm{ra}$, do là các tháng giữa và cuối mùa khô nên khô hạn trong thực tế thường xảy ra vào các tháng này. Như vậy trên khu vực nghiên cứu thì $\mathrm{K}$ và $\mathrm{AIu}$ phù hợp trong đánh giá hạn hán đối với sản xuất nông nghiệp và PNI phù hợp trong đánh giá hạn khí tượng.

Trừ các chỉ số $P N I, K$ và AIu, các chỉ số còn lại có thời gian hạn phân bố khá đều trong năm là do: 1) Các chỉ số đều được tính riêng cho từng tháng; 2) Nhóm các chỉ số SPI, CZI và SPEI có độ lớn được tính theo tần suất xuất hiện dựa trên các hàm phân bố; nhóm $\mathrm{EDI}$, Aiu và Ped có độ lớn được xác định dựa trên các công thức chuẩn hóa dữ liệu. Cách tính này đã làm cho thời gian hạn xuất hiện tương đối đều giữa các tháng. 


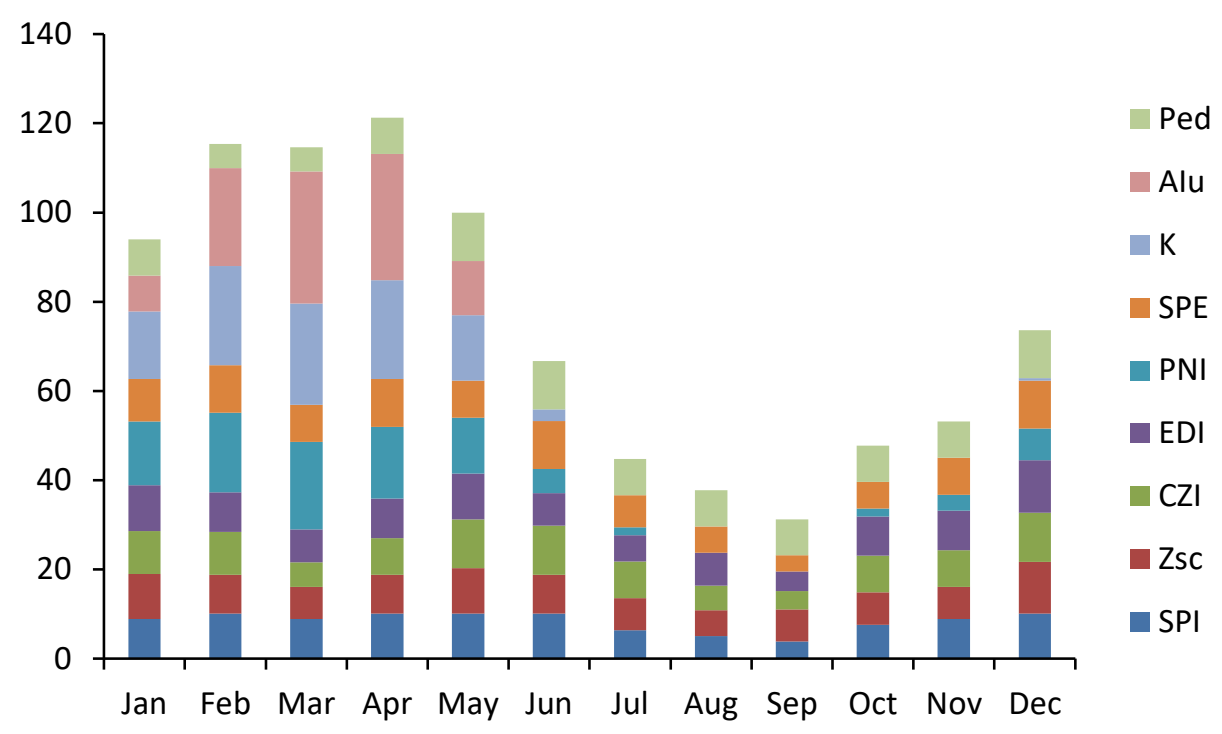

Hình 4. Thời gian xuất hiện hạn theo các tháng

Trên bảng 2 còn cho thấy thời gian khô hạn là không thống nhất giữa các chỉ số, với Zsc thời gian khô hạn chỉ chiếm $4,2 \%$ thời gian trong năm trong khi đó con số tương ứng với $\mathrm{AIu}$, PNI và là 23,6\%,24,8\% và $36,7 \%$. Về khả năng thể hiện mức hạn của các chỉ số SPI, CZI và ZSc phù hợp với nghiên của Morid và và cộng sự (2006), nghiên cứu này cũng chỉ ra rằng các chỉ số hạn này là phản ứng chậm với khởi phát hạn hán. Như vậy để sử dụng các chỉ số này trong đánh giá tình trạng khô hạn cần điều chỉnh lại thang đánh giá. Để thấy được mức độ phù hợp của các chỉ số trên cho SVN cũng như thấy đặc điểm hạn của khu vực này, dưới đây phân tích chỉ số hạn $\mathrm{K}$, đây là chỉ số hạn được sử dụng phổ biến ở Việt nam. Sử dụng $\mathrm{K}>1$ và thời gian kéo dài 2 tháng để xác định thời gian hạn, kết quả thống kê được trình bày trong Bảng 4 .

Bảng 4 cho thấy có sự khác biệt đáng kể về thời gian hạn giữa RG1 và hai vùng còn lại. Ở RG1, thời gian hạn bắt đầu từ Jan tới Aug, trong khi đó ở hai vùng còn lại thì từ Jun tới Aug không xuất hiện hạn, sự khác biệt này là do RG1 chịu ảnh hưởng của phơn do địa hình núi cao dãy Trường Sơn gây ra. Ranh giới giữa RG1 và RG2 có độ cao từ 600m tới $1200 \mathrm{~m}$ là nguyên nhân của sự khác biệt này. Vào mùa gió mùa tây nam, từ tháng 5 đến 9 , là mùa mưa của RG2 và RG3, thì RG1 lại khô hạn do hiệu ứng phơn của dãy Trường Sơn. Ảnh hưởng của địa hình đã làm cho RG1 có thời gian khô hạn chiếm tới 44,2\% thời gian trong năm, trong khi đó RG2 và RG3 chỉ có thời gian khô hạn tương ứng là 32,5\% và 34,1\%.

Bảng 4. Thời gian hạn tính theo chỉ số $\mathrm{K}$ theo các vùng

\begin{tabular}{ccccc}
\multirow{2}{*}{ Month } & \multicolumn{4}{c}{ Thời gian hạn $(\%)$ theo các vùng } \\
\cline { 2 - 5 } & RG1 & RG2 & RG3 & SVN \\
\hline Jan & 1,7 & 8,3 & 7,5 & 6,0 \\
Feb & 7,0 & 9,8 & 8,6 & 8,4 \\
Mar & 9,8 & 8,6 & 8,6 & 8,9 \\
Apr & 9,5 & 3,4 & 6,5 & 6,6 \\
May & 7,0 & 0,7 & 1,4 & 2,9 \\
Jun & 4,3 & 0,0 & 0,0 & 1,3 \\
Jul & 3,1 & 0,0 & 0,0 & 0,9 \\
Aug & 1,7 & 0,0 & 0,0 & 0,5 \\
Sep & 0,0 & 0,0 & 0,0 & 0,0 \\
Oct & 0,0 & 0,0 & 0,0 & 0,0 \\
Nov & 0,0 & 0,0 & 0,0 & 0,0 \\
Dec & 0,0 & 1,7 & 1,4 & 1,1 \\
Year & 44,2 & 32,5 & 34,1 & 36,7 \\
\hline
\end{tabular}


So sánh kết quả tính toán trong bảng 3 cho thấy $\mathrm{AIu}$ và $\mathrm{PNI}$ là 2 chỉ số phù hợp hơn với $\mathrm{K}$ về thời gian khô hạn, tuy nhiên mức chênh lệch là còn khá đáng kể. Thời gian khô hạn tính theo $\mathrm{K}$ cho SVN khoảng $36,7 \%$ thời gian trong năm, trong khi đó con số tương ứng với $\mathrm{AIu}$ và $\mathrm{PNI}$ tương ứng là $23,6 \%$ và $24,8 \%$. Như vậy cũng cần điều chỉnh thang phân loại hạn cho AIu và PNI sao cho phù hợp hơn với SVN và Bảng 4 có thể là cơ sở để điều chỉnh các chỉ số này.

\subsection{Mối quan hệ của các chỉ số hạn với các chỉ số giám sát ENSO}

- Hệ số tuơng quan sử dụng một bước thời gian trễ

Để thấy được ảnh hưởng của ENSO đến tình hạn hán qua các chỉ số hạn, hệ số tương quan giữa các chỉ số ENSO và chỉ số hạn được sử dụng như một thước đo trong đánh giá. Kết quả tính toán hệ số tương quan lớn nhất ứng với thời gian trễ giữa 2 nhóm chỉ số này được trình bày trong Bảng 5 và Bảng 6 .

Bảng 5. Hệ số tương quan giữa các chỉ số ENSO và chỉ số hạn

\begin{tabular}{cccccccccc} 
& SPI & Zsc & CZI & EDI & PNI & SPEI & K & AIu & Ped \\
\cline { 2 - 9 } ONI & $-0,42$ & $-0,42$ & $-0,42$ & $-0,38$ & $-0,39$ & $-0,45$ & 0,20 & $-0,20$ & 0,42 \\
MEI & $-0,46$ & $-0,45$ & $-0,45$ & $-0,42$ & $-0,40$ & $-0,48$ & 0,19 & $-0,16$ & 0,33 \\
SOI & 0,33 & 0,33 & 0,34 & 0,32 & 0,31 & 0,37 & $-0,18$ & 0,16 & $-0,29$ \\
JMA & $-0,36$ & $-0,36$ & $-0,36$ & $-0,33$ & $-0,31$ & $-0,38$ & 0,11 & $-0,13$ & 0,41
\end{tabular}

Bảng 6. Thời gian trễ tính bằng tháng giữa các chỉ số ENSO và chỉ số hạn

\begin{tabular}{lccccccccc} 
& SPI & Zsc & CZI & EDI & PNI & SPEI & K & AIu & Ped \\
\cline { 2 - 10 } ONI & 1 & 1 & 1 & 1 & 1 & 1 & 1 & 1 & 3 \\
MEI & 1 & 1 & 1 & 1 & 1 & 1 & 4 & 4 & 3 \\
SOI & 2 & 2 & 2 & 2 & 2 & 2 & 2 & 2 & 4 \\
JMA & 2 & 2 & 2 & 2 & 2 & 3 & 1 & 1 & 4
\end{tabular}

Dựa trên Bảng 5 cho thấy tính trung bình theo các chỉ số giám sát ENSO thì hệ số tương quan tăng theo thứ tự thì SOI, JMA, ONI và MEI. Tương tự, theo các chỉ số hạn thì hệ số tương quan tăng dần theo thứ tự $\mathrm{AIu}$, $\mathrm{K}, \mathrm{PN}, \mathrm{Ped}, \mathrm{EDI}, \mathrm{Zsc}, \mathrm{SPI}, \mathrm{ZCI}$ và SPEI. Tuy nhiên các hệ số tương quan là khá nhỏ, hệ số tương quan lớn nhất là giữa $\mathrm{MEI}$ và $S P E I$ cũng chỉ đạt $-0,48$. Xem xét về thời gian trễ cho thấy ứng với $|R|>0,4$ thường có thời gian trễ khoảng 1 tháng, kết hợp với hệ số tương quan thấp điều này cho thấy mức ý nghĩa trong cảnh báo hạn hán của chúng là không cao.

- Hệ số tưong quan sử dụng thời gian trễ theo tùng tháng

Theo Bảng 5 , trong số các chỉ số giám sát ENSO thì chỉ có ONI và MEI là có quan hệ tốt hơn với các chỉ số khô hạn nên nó được chọn lựa để đưa vào phân tích.

+ Giữa MEI và các chỉ số hạn

\begin{tabular}{|c|c|c|c|c|c|c|c|c|c|c|c|c|c|}
\hline DrI & Jan & Feb & Mar & Apr & May & Jun & Jul & Aug & Sep & Oct & Nov & Dec & $\begin{array}{c}\text { Mean } \\
|\mathrm{R}|\end{array}$ \\
\hline Zsc & $-0,38$ & $-0,30$ & $-0,55$ & $-0,65$ & $-0,68$ & $-0,61$ & $-0,50$ & $-0,12$ & $-0,20$ & $-0,39$ & $-0,41$ & $-0,49$ & 0,44 \\
\hline $\mathrm{CZI}$ & $-0,35$ & $-0,29$ & $-0,55$ & $-0,69$ & $-0,68$ & $-0,62$ & $-0,50$ & $-0,10$ & $-0,22$ & $-0,37$ & $-0,40$ & $-0,48$ & 0,44 \\
\hline EDI & $-0,35$ & $-0,34$ & $-0,57$ & $-0,63$ & $-0,64$ & $-0,39$ & $-0,37$ & $-0,03$ & $-0,20$ & $-0,49$ & $-0,40$ & $-0,36$ & 0,40 \\
\hline SPEI & $-0,39$ & $-0,37$ & $-0,56$ & $-0,69$ & $-0,69$ & $-0,65$ & $-0,55$ & $-0,18$ & $-0,26$ & $-0,39$ & $-0,40$ & $-0,49$ & 0,47 \\
\hline K & 0,42 & 0,47 & 0,66 & 0,71 & 0,78 & 0,65 & 0,50 & 0,06 & 0,20 & 0,47 & 0,54 & 0,42 & 0,49 \\
\hline AIu & $-0,43$ & $-0,30$ & $-0,51$ & $-0,65$ & $-0,69$ & $-0,65$ & $-0,57$ & $-0,36$ & $-0,34$ & $-0,45$ & $-0,50$ & $-0,56$ & 0,50 \\
\hline Ped & 0,58 & 0,59 & 0,72 & 0,79 & 0,79 & 0,69 & 0,65 & 0,36 & 0,21 & $-0,12$ & 0,43 & 0,55 & 0,54 \\
\hline
\end{tabular}

Hệ số tương quan giữa MEI và một số các chỉ số khô hạn theo từng tháng được minh họa trên Hình 5 . Trong hình này các điểm có ký hiệu '+' là nơi có hệ số tương quan lớn nhất $\left(\mathrm{R}_{\max }\right)$ theo tháng tương ứng, 
hay nó thể hiện thời gian trễ của từng tháng. Từ các điểm có $\mathrm{R}_{\max }$, giá trị liệt kê của các điểm này được thể hiện trong Bảng 7 và Bảng 8 .

Bảng 7. Hệ số tương quan lớn nhất giữa MEI và các chỉ số khô hạn.

Bảng 8. Thời gian trễ ứng với hệ số tương quan lớn nhất giữa MEI và các chỉ số khô hạn.

\begin{tabular}{ccccccccccccc} 
DrI & Jan & Feb & Mar & Apr & May & Jun & Jul & Aug & Sep & Oct & Nov & Dec \\
\hline SPI, Zsc, CZI, EDI, & 1 & 1 & 3 & 3 & 3 & 5 & 5 & 6 & 6 & 1 & 1 & 1 \\
PNI, SPEI, K & 1 & & & & & & & & & & & \\
PED & 2 & 6 & 5 & 4 & 3 & 3 & 4 & 5 & 6 & 5 & 5 & 2
\end{tabular}

Bảng 8 và Bảng 9 cho thấy không có sự khác biệt về mối quan hệ giữa MEI với các chỉ số SPI, Zsc, CZI, PNI, K và AIu. Các chỉ số này đều có quan hệ rõ rệt hơn với MEI trong khoảng từ tháng 4 tới tháng 6 với hệ số tương quan cao nhất khoảng $0,68-0,78$ và xảy ra vào tháng 5 . Trong số các chỉ số khô hạn này thì EDI có mối quan hệ kém nhất với MEI và ngược lại đối với $P E D$. Hệ số tương quan cao nhất giữa MEI và PED xảy ra từ tháng 3 tới tháng 7 với $R$ thường trên 0,65 , và cao nhất là 0,79 vào tháng 4 và tháng 5 . Về thời gian trễ, PED có thời gian trễ so với MEI từ 2 đến 6 tháng. Các chỉ số còn lại có chung một thời gian trễ, từ tháng 10 tới tháng 2 thì thời gian trễ là 1 tháng, các tháng còn lại có thời gian trễ từ 3 tới 6 tháng.
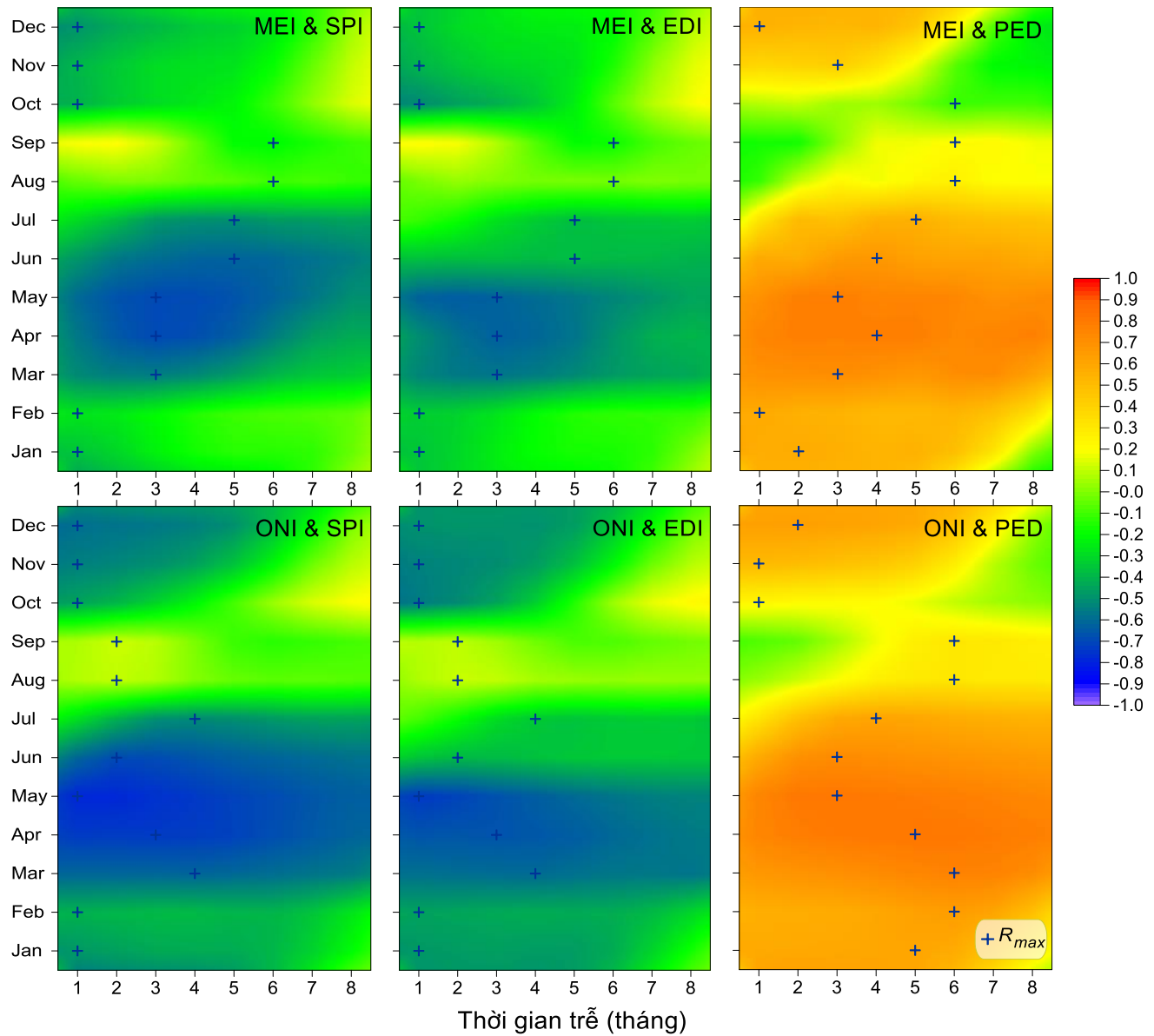

Hình 5. Hệ số tương quan giữa $\mathrm{SPI}$, EDI và $\mathrm{PED}$ với $\mathrm{MEI}$ và $\mathrm{ONI}$ tính cho từng tháng

Như vậy nếu sử dụng MEI để cảnh báo khô hạn thông qua nhóm các chỉ số có liên quan đến lượng mưa hoặc lượng mưa và lượng bốc thoát hơi thì chỉ phù hợp cho các tháng từ tháng 4 tới tháng 6 với thời gian cảnh báo trước (thời gian trễ) từ 3 đến 5 tháng. Với chỉ số liên quan đến nhiệt độ và lượng mưa, Ped, có thể cảnh báo khô hạn theo chỉ số này cho các tháng 3 tới tháng 7 với thời gian cảnh báo trước từ 3 đến 5 tháng. Mối quan hệ giữa MEI và PED là tốt hơn so với các chỉ số khác vì trong thành phần của PED chứa yếu tố nhiệt độ, theo Lương (2006) thì mối quan hệ giữa MEI và nhiệt độ khu vực nghiên cứu là khá tốt.

\section{+ Giữa ONI và các chỉ số hạn}

Tương tự với MEI, hệ số tương quan với các chỉ số khô hạn cũng được tính toán, Hình 5 là minh họa về 
tương quan giữa $\mathrm{ONI}$ và một số chỉ số khô hạn. Dựa trên kết quả tính toán này giá trị $\mathrm{R}$ lớn nhất cùng thời gian trễ tương ứng được trình bày trong Bảng 9 và Bảng 10 .

Bảng 9 . Hệ số tương quan lớn nhất giữa $\mathrm{ONI}$ và các chỉ số khô hạn.

\begin{tabular}{lccccccccccccc} 
DrI & Jan & Feb & \multicolumn{1}{c}{ Mar } & \multicolumn{1}{c}{ Apr } & \multicolumn{1}{c}{ May } & \multicolumn{1}{c}{ Jun } & \multicolumn{1}{c}{ Jul } & Aug & \multicolumn{1}{c}{ Sep } & \multicolumn{1}{c}{ Oct } & Nov & Dec & \multicolumn{2}{c}{ Mean } \\
\hline SPI & $-0,48$ & $-0,41$ & $-0,62$ & $-0,74$ & $-0,79$ & $-0,70$ & $-0,52$ & 0,10 & $-0,14$ & $-0,45$ & $-0,55$ & $-0,60$ & 0,51 \\
Zsc & $-0,50$ & $-0,42$ & $-0,62$ & $-0,70$ & $-0,78$ & $-0,68$ & $-0,51$ & 0,08 & 0,13 & $-0,44$ & $-0,55$ & $-0,60$ & 0,50 \\
CZI & $-0,48$ & $-0,42$ & $-0,62$ & $-0,73$ & $-0,79$ & $-0,70$ & $-0,52$ & 0,09 & $-0,17$ & $-0,44$ & $-0,55$ & $-0,59$ & 0,51 \\
EDI & $-0,48$ & $-0,45$ & $-0,61$ & $-0,67$ & $-0,74$ & $-0,39$ & $-0,37$ & 0,11 & 0,10 & $-0,56$ & $-0,55$ & $-0,50$ & 0,46 \\
PNI & $-0,50$ & $-0,41$ & $-0,60$ & $-0,69$ & $-0,78$ & $-0,70$ & $-0,53$ & $-0,05$ & $-0,15$ & $-0,49$ & $-0,60$ & $-0,65$ & 0,51 \\
SPEI & $-0,52$ & $-0,50$ & $-0,66$ & $-0,74$ & $-0,80$ & $-0,72$ & $-0,58$ & $-0,14$ & $-0,19$ & $-0,44$ & $-0,53$ & $-0,60$ & 0,54 \\
K & 0,32 & 0,36 & 0,60 & 0,69 & 0,74 & 0,65 & 0,45 & 0,16 & 0,20 & 0,54 & 0,50 & 0,41 & 0,48 \\
AIu & $-0,55$ & $-0,42$ & $-0,61$ & $-0,70$ & $-0,79$ & $-0,74$ & $-0,58$ & $-0,28$ & $-0,25$ & $-0,44$ & $-0,63$ & $-0,66$ & 0,55 \\
Ped & 0,61 & 0,65 & 0,76 & 0,82 & 0,81 & 0,73 & 0,66 & 0,31 & 0,30 & 0,23 & 0,52 & 0,64 & 0,59
\end{tabular}

Bảng 10. Thời gian trễ ứng với hệ số tương quan lớn nhất giữa ONI và các chỉ số khô hạn.

\begin{tabular}{ccccccccccccc} 
DrI & Jan & Feb & Mar & Apr & May & Jun & Jul & Aug & Sep & Oct & Nov & Dec \\
\hline SPI, Zsc, CZI, EDI, & 1 & 1 & 3 & 3 & 3 & 5 & 5 & 6 & 6 & 1 & 1 & 1 \\
PNI, SPEI, K & 1 & & & & & & & & & & & \\
PED & 5 & 6 & 6 & 5 & 3 & 3 & 4 & 6 & 6 & 1 & 1 & 2
\end{tabular}

So sánh giữa bảng 8 và bảng 10 cho thấy có sự gia tăng nhẹ của $R$ khi thay thế $\mathrm{MEI} \mathrm{bằng} \mathrm{ONI}$. Trong bảng 10, tính trung bình thì hệ số tương quan tăng dần theo các chỉ số sau EDI, K, ZSc, cặp (SPI, CZI), PNI, SPEI, AIu và PED. Như vậy sử dụng ONI trong giám sát hạn hán là tốt hơn so với MEI.

\section{KẾT LUẬN}

Qua kết quả phân tích các chỉ số khô hạn nêu trên cho thấy:

- Với các chỉ số khô hạn được xây dựng dựa trên 1 yếu tố là lượng mưa thì PN là một chỉ số thể hiện được tình hình khô hạn phù hợp với phân bố của lượng mưa trong năm. Ngoài ra đây là một chỉ số khá đơn giản trong tính toán và có quan hệ tốt với ONI. Tuy nhiên nếu phân tích rời rạc cho từng tháng thì có thể sử dụng SPI hoặc CZI vì nó cũng có quan hệ tốt với ONI, tuy nhiên cần điều chỉnh thang phân loại cấp hạn của các chỉ số này sao cho phù hợp với tình hình khô hạn trong thực tế.

- Với các chỉ số khô hạn được xây dựng dựa trên lượng mưa và lượng bốc thoát hơi thì Alu là một lựa chọn vì nó khá phù hợp với tình hìn khô hạn khu vực nghiên cứu và cũng có quan hệ tốt với ONI. Như vậy Alu khá phù hợp trong đánh giá hạn hán đối với sản xuất nông nghiệp cũng như cảnh báo hạn hán do tác động của ENSO, mặc dù vậy vẫn cần phải điều chỉnh thang phân loại hạn của chỉ số này để nó phù hợp với khu vực nghiên cứu. Trong nhóm các chỉ số này thì $\mathrm{K}$ là chỉ số được sử dụng chủ yếu ở nước ta, chỉ số $\mathrm{K}$ là nghịch đảo của $\mathrm{AIu}$ vì vậy nó có nhiều tính chất chung, tuy nhiên mức độ nhạy cảm của $\mathrm{K}$ với ENSO là thấp hơn.

- Đối với chỉ số khô hạn dựa trên nhiệt độ và lượng mưa, Ped, đây là một chỉ số thể hiện rõ nét nhất các ảnh hưởng của ENSO. Chỉ số này có ưu điểm là ngoài thể hiện tình trạng khô hạn nó còn thể hiện tình trạng nắng nóng. Tuy nhiên chỉ số này chỉ phù hợp khi phân tích tình hình khô hạn rời rạc cho từng thời gian trong năm và cần điều chỉnh thang phân loại khô hạn cho phù hợp với khu vực nghiên cứu.

Ngoài ra kết quả nghiên cứu này còn cho thấy ENSO chỉ tác động đến hạn hán khu vực này rõ rệt trong một số tháng, rõ nét nhất là từ tháng 3 đến tháng 6 . Trong số chỉ số giám sát ENSO đưa vào phân tích thì ONI là chỉ số nhạy nhất với tình hình khô hạn khu vực nghiên cứu.

\section{LỜI CẢM ƠN}

Kết quả nghiên cứu này được sự hỗ trợ từ dự án 2.21 về giám sát hạn hán cho Việt Nam, đây là dự án trong khuôn khổ hợp tác song phương giữa Việt Nam và Chính phủ Wallonie-Bruxelles trong giai đoạn 20192021. Chúng tôi xin chân thành cảm ơn các tổ chức liên quan đến dự án này. 


\section{TÀI LIỆU THAM KHẢO}

[1] Anli, A.S., 2017. Temporal variation of reference evapotranspiration and regional drought estimation using SPEI method for semi-arid Konya closed basin in Turkey. European Water, 59, 231-238.

[2] Byun HR, Wilhite DA., 1999. Objective quantification of drought severity and duration. Journal of Climate, $12,2747-2756$.

[3] Chen T.C., Tsay J.D., Yen M.C., Matsumoto J., 2012. Interannual variation of the late fall rainfall in Central Vietnam. J. Clim., 25(1), 392-413, doi: 10,1175/JCLI-D-11-00068,1.

[4] Gobin A., Nguyen H. T., Pham V. Q. and Pham H. T. T., 2015. Heavy rainfall patterns in Vietnam and their relation with ENSO cycles. Int. J. Climatol., 14, doi: 10,1002/joc,4451.

[5] Ha N.T.T., Nhuan M.T., Canh B.D., Thao N.T.P., 2016. Mapping Droughts Over the Central Highland of Vietnam in El Niño Years Using Landsat Imageries. VNU J. Science, 32, 255-263.

[6] Holly C. Y. L., Andy H., Glenn MG, Emily Y. Y. C. and Shakoor H., 2019.Time-Series Study of Associations between Rates of People Affected by Disasters and the El Niño Southern Oscillation (ENSO) Cycle. Int. J. Environ. Res., 3146, 16, doi:10,3390/ijerph16173146.

[7] Lương Văn Việt, 2006. ENSO và khả năng dự báo mưa, nhiệt, ẩm cho Nam Bộ. Tạp chí Khí tượng Thủy văn, $551,39-45$.

[8] Mai T.T. and Hoang L. T. T., 2017. The relationship between rainfall over Vietnam and ENSO indices. J. Hydrometeorology, 553, 13-23.

[9] Mckee T. B., Doesken N. J. and Kleist J., 1993. The relationship of drought frequency and duration to time scale. Proceedings of the Eighth Confrence on Applied Climatology, American Meteorological Society: Boston, 179-184.

[10] Morid, Saeid \& Smakhtin, Vladimir \& Moghaddasi, Mahnosh, 2006. Comparison of seven meteorological indices for drought monitoring in Iran. International Journal of Climatology, 26, 971-985, doi:10,1002/joc, 1264 .

[11] Nguyen D.-Q., et al., 2014. Variations of surface temperature and rainfall in Vietnam from 1971 to 2010. Int. J. Climatol., 34, 249-264, doi: 10,1002/joc,3684.

[12] Nguyen T.V., Mai V.K. et al., 2019. Evaluation of summer monsoon climate predictions over the Indochina Peninsula using regional spectral model. J. Weather Clim. Extrem, 23, 14 doi: 10,1016/j.wace,2019,100195.

[13] Pedey, D.A, 1975. On indicators of droughts and wet conditions. Proc. USSR Hydrometeorol. Centre Russian Meteorol. Hydrol., 156, 19-39.

[14] United Nations Educational, Scientific and Cultural Organization (UNESCO), 1979. Map of the World Distribution of Arid Regions: Map at Scale 1:25,000,000 with Explanatory Note; MAB Technical Notes 7; UNESCO: Paris, France.

[15] Vicente-Serrano, S. M., Begueria, S. and Lopez-Moreno, J. I., 2010. A multiscalar drought index sensitive to global warming: The SPEI. Journal of Climate, 23: 1696-1718.

[16] Vinit K. Jain et al., 2015. Comparison of drought indices for appraisal of drought characteristics in the Ken River Basin. Weather and Climate Extremes, 8, 1-11, 29, doi: 10,1016/j.wace,2015,05,002.

[17] Willeke, G., Hosking, J.R.M., Wallis, J.R., Guttman, N.B, 1994. The national drought atlas. Institute for Water Resources Report 94-NDS-4, U.S. Army Corps of Engineers.

[18] World Meteorological Organization (WMO) and Global Water Partnership (GWP), 2016. Handbook of Drought Indicators and Indices. Geneva, Switzerland, 2016; 1-45.

[19] Wu, H., Hayes, M.J., Weiss, A., Hu, Q.I., 2001. An evaluation of the standardized precipitation index, the china-Zindex and the statistical Z-Score. Int. J. Climatol., 21, 745-758, doi:10,1002/joc,658. 